\title{
Effect of functionalization and size of CNTs in the production of nanocomposites
}

\author{
Sónia Simões ${ }^{1}$, Paulo J. Ferreira ${ }^{2,3}$, Filomena Viana ${ }^{1}$, Marcos A.L. Reis ${ }^{4}$ and Manuel F. Vieira ${ }^{1}$ \\ 1.CEMMPRE, Department of Metallurgical and Materials Engineering, University of Porto, R. Dr. \\ Roberto Frias, 4200-465 Porto, Portugal \\ 2. Materials Science and Engineering Program, University of Texas at Austin, Austin, TX, 78712, USA \\ ${ }^{3}$ International Iberian Nanotechnology Laboratory, Avenida Mestre José Veiga, 4715-330 Braga, \\ Portugal \\ 4.Faculdade de Ciências Exatas e Tecnologia, Universidade Federal do Pará, Abaetetuba, PA 68440-000 \\ Brazil
}

Metal matrix composites (MMCs) generally offer a wide range of properties, such as high specific strength stiffness and good wear resistance. Carbon-based nanomaterials, in particular carbon nanotubes (CNTs), graphene or nanodiamonds, became very attractive as reinforcements for MMCs. Specifically, CNTs have received great attention from the research community due to their extraordinary properties, such as high stiffness, high strength and high thermal conductivity, combined with their low weight [1]. However, the dispersion of these nanofillers remains to be a major challenge during the production of the nanocomposites [2,3]. In this context, functionalization of CNTs may promote a better dispersion, but the mechanical properties of the composites may be impaired by damage of CNTs during the process. Several studies have been carried out to develop new techniques to obtain a good and uniform dispersion of CNTs throughout the matrix. Nevertheless, there are few studies analyzing the effect of length, outer diameter and surface modification of CNTs on the production of MMCs.

In this context, the focus of this work is the study of the effect of size and functionalization of CNTs in the production of Al/CNTs nanocomposites. Prior to this work, ultrasonication was used to simultaneously disperse the CNTs and mixture them with Al powders. This approach proved to be a good option to produce Al/CNTs nanocomposites with a significant increase in mechanical properties [3]. However, the use of different CNTs, especially those that are functionalized, can improve the dispersion and promote the production of nanocomposites with better mechanical properties.

The production of $\mathrm{Al} / \mathrm{CNT}$ nanocomposites was performed by powder metallurgy using ultrasonication to disperse and mix CNTs and Al powders. The Al powders (from Goodfellow) exhibit a D50 of $60 \mu \mathrm{m}$ with a purity of $99.5 \%$. Three different types of multi-walled carbon nanotubes (MWCNTs) were used: two types of MWCNTs from Nanothinx S.A (non-functionalized and functionalized) with a purity of $95 \%$, outer diameters ranging from 15 to $35 \mathrm{~nm}$ and lengths of approximately $10 \mu \mathrm{m}$ and MWCNTs from Nanocyl, which were finer (average outer diameter of $9.5 \mathrm{~nm}$ ) and shorter $(1.5 \mu \mathrm{m})$. The characterization of the MWCNTs was performed by scanning and transmission electron microscopy (SEM and TEM). MWCNTS were dispersed and mixed with Al using an ultrasonicator during 15 minutes in isopropanol. The compacts, produced with $0,0.5,0.75$ and $1 \mathrm{wt} . \%$ of CNTs, were sintered at $640{ }^{\circ} \mathrm{C}$ during 90 min under a vacuum greater than $10^{-2} \mathrm{~Pa}$. Microstructural characterization of these nanocomposites was performed by optical microscopy (OM), SEM, TEM and electron backscattered diffraction (EBSD). The mechanical properties were evaluated by Vickers microhardness tests using a $98 \mathrm{mN}$ load; ten tests have been performed on each sample. 
The microstructural characterization of the MWCNTs revealed that the surface modification of the CNTs induces significant damage to the structure of the CNTs. Figure 1 shows the TEM images of the nonfunctionalized and functionalized MWCNTs. These images show that the functionalized MWCNTs exhibit some defects at the walls, smaller diameters and shorter lengths that result from the functionalization treatment.

Microstructural characterization of nanocomposites revealed that the CNTs dispersion is strongly affected by the size and surface modifications of the CNTs. The use of functionalized CNTs or thin CNTs allowed the production of composites with a better dispersion of CNTs and with smaller CNTs agglomerates.

\section{References}

[1] K.U. Kainer, WILLEY-VGH Verlag GmbH \& Co. KGaA. Weinheim, 2006

[2] O. Carvalho, G. Miranda, D. Soares and F. S. Silva, Mechanics of Advanced Materials and Structures 23 (2016), p. 66.

[3] S. Simões, F. Viana, M.A.L. Reis and M.F. Vieira, Composites Structures 108 (2014) p. 992.

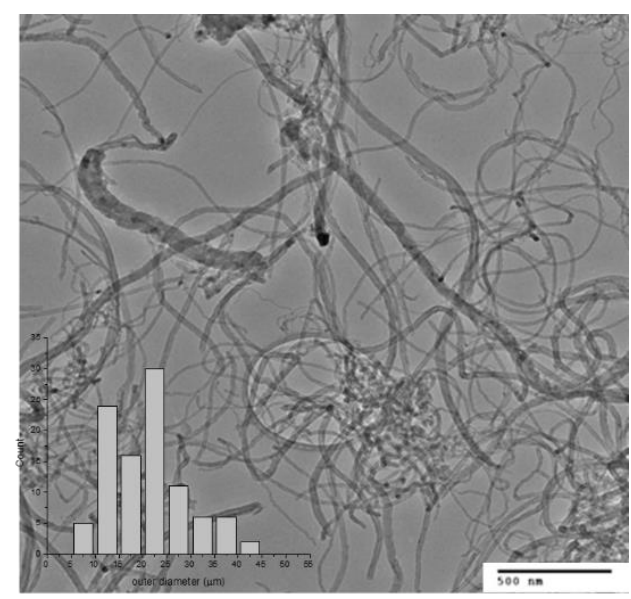

(a)

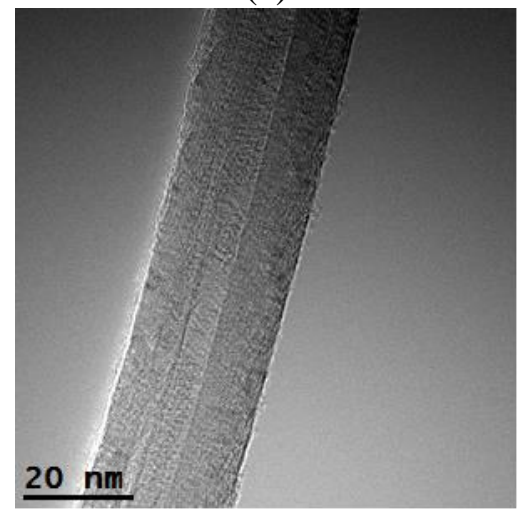

(c)

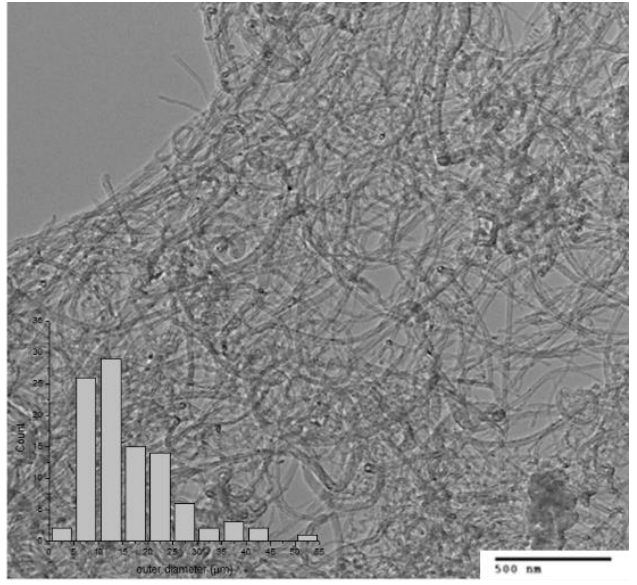

(b)

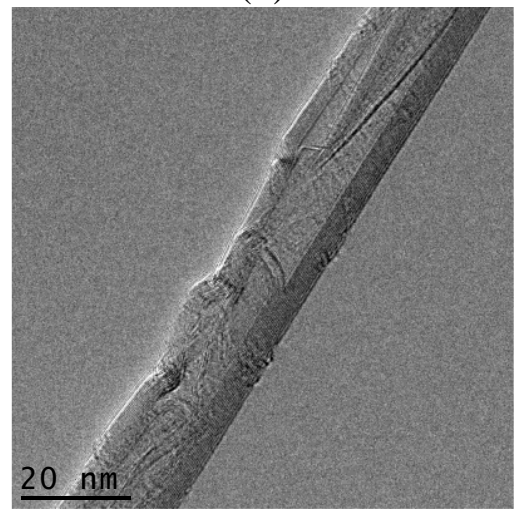

(d)

Figure 1. Bright-field TEM images ( $a$ and $b$ ) and phase contrast TEM images (c and d) of nonfunctionalized MWCNTS (a and $c$ ) and functionalized MWCNTS (b and d). The outer diameter distributions of MWCNTs can also be observed in a) and b). 